IMA Journal of Applied Mathematics (2019) Page 1 of 17 doi:10.1093/imamat/xxx000

\title{
Reflection and transmission of high-frequency acoustic, electromagnetic, and elastic waves at a distinguished class of irregular, curved boundaries
}

\author{
A. M. R. RADJEN* \\ School of Mathematical Sciences, University of Nottingham, University Park, Nottingham, \\ NG7 2RD, UK*Corresponding author: Anthony.Radjen@Nottingham.ac.uk \\ G. GRADONI \\ School of Mathematical Sciences, University of Nottingham, University Park, Nottingham, \\ $N G 72 R D, U K$ \\ AND \\ R. H. TEW \\ School of Mathematical Sciences, University of Nottingham, University Park, Nottingham, \\ $N G 72 R D, U K$
}

[Received on 5 November 2019]

\begin{abstract}
Reflection and transmission phenomena associated with high-frequency linear wave incidence on irregular boundaries between adjacent acoustic or electromagnetic media, or upon the irregular free surface of a semi-infinite elastic solid, are studied in two dimensions. Here, an "irregular" boundary is one for which small-scale undulations of an arbitrary profile are superimposed upon an underlying, smooth curve (which also has an arbitrary profile), with the length scale of the perturbation being prescribed in terms of a certain inverse power of the large wave-number of the incoming wave field. Whether or not the incident field has planar or cylindrical wave-fronts, the associated phase in both cases is linear in the wave-number, but the presence of the boundary irregularity implies the necessity of extra terms, involving fractional powers of the wave-number in the phase of the reflected and transmitted fields. It turns out that there is a unique perturbation scaling for which precisely one extra term in the phase is needed and hence for which a description in terms of a Friedlander - Keller ray expansion in the form as originally presented is appropriate, and these define a "distinguished" class of perturbed boundaries and are the subject of the current paper.
\end{abstract}

Keywords: Ray Theory, WKBJ-method, Wave Scattering

2000 Math Subject Classification: 34K30, 35K57, 35Q80, 92D25

\section{Introduction}

The problems which are addressed in this paper involve acoustic, electromagnetic, and elastic wave propagation, although there are fundamental features common to all three of these situations.

First, an oscillatory time-dependence $e^{-i \omega t}$ is always assumed but suppressed, so that if the wavespeed within the medium in question is $c$ then the relevant field equation is the two-dimensional Helmholtz equation

$$
\left(\frac{\partial^{2}}{\partial x^{2}}+\frac{\partial^{2}}{\partial y^{2}}+k^{2}\right) \phi=0
$$


in which $k=\frac{\omega}{c}$ is the wave-number (so that $\frac{2 \pi}{k}$ is the associated wave-length), and $\phi$ is the relevant field variable (such as a pressure field, displacement potential function, or a component of an electric/magnetic field, respectively, in each of the three applications referred to above).

Second, relative to the same Cartesian coordinates $(x, y)$ used in the Helmholtz equation (1.1), the domain occupied by this medium is $\hat{D}$, and is bound by the smooth curve $\partial \hat{D}$ which is given parametrically as

$$
\partial \hat{D}: \quad \boldsymbol{x}=\boldsymbol{x}_{0}(s)+k^{-1 / N} \hat{\boldsymbol{x}}_{0}(s),
$$

where $\boldsymbol{x}=(x, y)$, with $\partial D$ being the corresponding $O(1)$ boundary given by $\boldsymbol{x}=\boldsymbol{x}_{0}$. Thus, $\hat{\boldsymbol{x}}_{0}$, which is an $O(1)$ variable, denotes the small-scale undulations imposed onto $x_{0}$, and so the domain above is defined by $y>y_{0}(s)+k^{-1 / N} \hat{y}_{0}(s)$. The variable $N$ will actually be specified to be $N=2$ shortly, but it is helpful to leave $N$ as a general positive integer for the purposes of this introduction. In both the acoustic and the electromagnetic cases, the region $y<y_{0}(s)+k^{-1 / N} \hat{y}_{0}(s)$ is taken to be occupied by a second "lower" medium for which the Helmholtz equation (1.1) is to be replaced by

$$
\left(\frac{\partial^{2}}{\partial x^{2}}+\frac{\partial^{2}}{\partial y^{2}}+\frac{k^{2}}{\gamma^{2}}\right) \phi=0
$$

where $\frac{1}{\gamma}$ (the refractive index in optical terms) is a given positive $O(1)$ constant. Also, the parameter $s$ occurring in boundary (1.2) is taken to be the arc-length measured along the curve $x=x_{0}(s), y=y_{0}(s)$ (so that $\left(x_{0}^{\prime}\right)^{2}+\left(y_{0}^{\prime}\right)^{2}=1$, a result that is used repeatedly in the applications that follow). Notice that appropriate interfacial/boundary conditions need to be imposed along boundary (1.2), and these shall be specified for each class of problems in turn.

Third, the wave-number $k$ occurring in both Helmholtz equations (1.1) and (1.3) is large, in which case they are both singularly-perturbed partial differential equations amenable to WKBJ-type analysis, whilst the term proportional to $k^{-1 / N}$ on the right-hand side of boundary (1.2) can be interpreted as a small perturbation of scale $O\left(k^{-1 / N}\right)$ of profile $\left(\hat{x}_{0}(s), \hat{y}_{0}(s)\right)$ superimposed on the underlying curve $\boldsymbol{x}=\left(x_{0}(s), y_{0}(s)\right)$.

In summary, the problems have two wave-bearing media both supporting high-frequency, short waves and are separated by a smooth, curved interface superimposed on which are small-scale undulations; the elastic case has the second "lower" medium replaced by a vacuum. The plan is to calculate the effects that these boundary perturbations have on the reflected and (if applicable) the transmitted fields when subject to specified wave incidence from the "upper" medium. Then the study of $(i)$ transmission problems at the common interface between adjacent acoustic wave-bearing media and (ii) applications in electromagnetism and elasticity are considered, with these applications being natural and significant extensions to those considered previously by Tew $(2018,2019)$.

Finally, referring to Tew $(2018,2019)$ once again, the case $N=2$ is distinguished compared to any other value $N=3,4,5, \cdots$. Without repeating in full the arguments presented there, an incident plane wave of the form

$$
\phi_{p}^{(i n c)}(x, y) \sim F_{p}(x \tan (\theta)+y) \exp (i k[x \cos (\theta)-y \sin (\theta)]),
$$


(which is an asymptotic solution of the Helmholtz equation (1.1) as $k \rightarrow \infty$ with $F_{p}$ being arbitrary to specify the incident amplitude profile, and $\theta$ is the angle of incidence between the ray and the $x$-axis) necessarily has terms proportional to $k^{(N-1) / N}\left(\hat{x}_{0}(s) \cos (\theta)-\hat{y}_{0}(s) \sin (\theta)\right)$ in its phase when evaluated on the boundary (1.2), in addition to the standard term $k\left(x_{0}(s) \cos (\theta)-y_{0}(s) \sin (\theta)\right)$. For an incident cylindrical wave of the form $\phi_{c}^{(i n c)}$, which is emitted from a point source located at $\boldsymbol{x}=(0, h)$,

$$
\phi_{c}^{(i n c)} \sim \frac{F_{c}(\Theta)}{\sqrt{R}} \exp (i k R)
$$

is also an asymptotic solution of the Helmholtz equation (1.1) as $k \rightarrow \infty$; where $R=\left(x^{2}+(y-h)^{2}\right)^{1 / 2}$, $\Theta$ is the polar angle from the point source, and $F_{c}$ denoted the angular directivity of the cylindricallyspreading wave field. The Taylor series expansion of $R$ along the boundary (1.2) has all values $O\left(k^{m / N}\right)$, $m=0,1, \cdots, N$. In either case, all of these extra terms (i.e. in addition to the $O(k)$ ) are inherited by the phases of the reflected and transmitted fields, necessitating solutions for these quantities of the general form

$$
\sum_{n=0}^{\infty} \frac{A_{n}(x, y)}{k^{n / N}} \exp \left(i \sum_{m=1}^{N} k^{(N+1-m)) / N} v_{m}(x, y)\right)
$$

and so not of the form $\exp \left(i k u(x, y)+k^{\alpha} v(x, y)\right) \sum_{n=0}^{\infty} \frac{A_{n}(x, y)}{k^{\lambda_{n}}}$, with just two terms in the exponent, one proportional to $k$ and one other as discussed by Friedlander and Keller (1955). Hence, their pioneering theory has to be amended in order to make it applicable here.

Considering the $m=0$ term in the various phase terms of $O\left(k^{m / N}\right)$ just mentioned as being more accurately described as part of the amplitude pre-factor to the exponential in the ansatz then the only instance that an $O(k)$ term and precisely one other term appear in the phase is when $N=2$, corresponding to a term proportional to $k^{1 / 2}$ in the exponent. This corresponds to setting $\alpha=\frac{1}{2}$ into the ansatz proposed by Friedlander and Keller and coincides precisely with the unique value of $\alpha$ that they identity as being distinguished form all others. Additionally, a separate geometrical argument first put forward by Engineer et al (1998) also identified $N=2$ as an isolated case of special importance, and so this value is substituted into boundary (1.2) from now onwards.

\section{The Friedlander-Keller ray expansion}

With the value of $N=2$ highlighted as a special case, the ansatz

$$
\phi(\boldsymbol{x})=\exp \left(i k u(\boldsymbol{x})+i k^{1 / 2} v(\boldsymbol{x})\right) \sum_{n=0}^{\infty} \frac{A_{n}(\boldsymbol{x})}{k^{n / 2}} .
$$

is taken. Substitution of this ansatz into the Helmholtz equation (1.1) reveals that $u$ obeys the eikonal equation

$$
\nabla u \cdot \nabla u=1 .
$$

The second-order equation then yields the governing equation for $v$ as

$$
\nabla u \cdot \nabla v=0 .
$$


The lower order terms give the family of recursive transport equations for the amplitude functions $A_{n}$

$$
\begin{aligned}
2 \boldsymbol{\nabla} A_{0} \cdot \boldsymbol{\nabla} u+A_{0}\left[\nabla^{2} u+i \boldsymbol{\nabla} v \cdot \nabla v\right] & =0, \\
2\left[\boldsymbol{\nabla} A_{n} \cdot \boldsymbol{\nabla} u+\nabla A_{n-1} \cdot \nabla v\right]-i \nabla^{2} A_{n-2}+A_{n-1} \boldsymbol{\nabla}^{2} v+A_{n}\left[\nabla^{2} u+i \boldsymbol{\nabla} v \cdot \nabla v\right] & =0,
\end{aligned}
$$

where $n=1,2,3, \cdots$ and $A_{-1}=0$ is understood when taking $n=1$ in equation (2.5).

The first step is to solve the eikonal equation (2.2) using Charpit's method of characteristics (which will become the "rays" here), as described by Zauderer (1989), Ockendon et al (2003), and applied by Tew (2019). Defining $\boldsymbol{p}=\boldsymbol{\nabla} u$, the idea is to introduce curves $\Gamma(s, \tau)$, parametrised by arc-length $\tau$ and emanating from the boundary $\boldsymbol{x}=\boldsymbol{x}_{0}(s)$, defined by $\frac{d \boldsymbol{x}}{d \tau}=\boldsymbol{p}$. The eikonal equation then reduces to $\frac{d u}{d \tau}=1$ along $\Gamma(s, \tau)$, so that if measuring $\tau$ from $\partial D$ and if $u=u_{0}(s)$ on $\partial D$ (so that $u=u_{0}(s)$ when $\underset{\tau}{d}=0$ ), the ray solution for $u$ is in the form

$$
u(s, \tau)=u_{0}(s)+\tau, \quad \text { along } \Gamma(s, \tau) .
$$

It is not difficult to show that $\boldsymbol{p}$ - which defines the directions of the rays - is constant along them so that $\boldsymbol{p}=\boldsymbol{p}_{0}(s)=\left(p_{0}(s), q_{0}(s)\right)$ along $\Gamma(s, \tau)$. This, in turn, allows the defining equation $\frac{d \boldsymbol{x}}{d \tau}=\boldsymbol{p}$ to be integrated, allowing the rays to be identified as the family of straight lines

$$
\Gamma(s, \tau): \boldsymbol{x}=\boldsymbol{x}_{0}(s)+\tau \boldsymbol{p}_{0}(s),
$$

and all that remains to do is calculate $p_{0}$ and $q_{0}$. The first of two equations for these is the eikonal equation itself, in the form

$$
p_{0}^{2}+q_{0}^{2}=1
$$

and the second is obtained by differentiation of the boundary data $u_{0}(s)$ (which is assumed to be prescribed or calculable) to give

$$
u_{0}^{\prime}=x_{0}^{\prime} p_{0}+y_{0}^{\prime} q_{0} .
$$

These are solved in terms of the local tangent vector $\boldsymbol{t}=\left(x_{0}^{\prime}, y_{0}^{\prime}\right)$ and normal vector $\boldsymbol{n}=\left(-y_{0}^{\prime}, x_{0}^{\prime}\right)$ to give

$$
\boldsymbol{p}_{0}=u_{0}^{\prime} \boldsymbol{t}(s) \pm \boldsymbol{n}(s) \sqrt{1-\left(u_{0}^{\prime}\right)^{2}}
$$

where the ambiguity in sign is resolved in any particular instance by requiring that any scattered rays obey the radiation condition and propagate away from the boundary.

Noting also that since $\nabla u=\frac{d x}{d \tau}$ along $\Gamma(s, \tau)$, equation (2.3) implies that $\frac{d v}{d \tau}=0$, so that $v$ is invariant along the rays, i.e.

$$
v(s, \tau)=v_{0}(s)
$$


where $v_{0}(s)$ is the value of $v$ on $\partial D$.

Calculating $\nabla^{2} u$ and $\nabla v$ in terms of the ray coordinates $(s, \tau)$ shows that $A_{0}$ satisfies the first-order differential equation

$$
2 \frac{\partial A_{0}}{\partial \tau}+A_{0}(s, \tau)\left[\frac{1}{\tau+a}+\frac{i b}{(\tau+a)^{2}}\right]=0,
$$

along the rays $\Gamma$. This is easily solved and has the solution

$$
A_{0}(s, \tau)=A_{0}(s, 0) \sqrt{\frac{a}{\tau+a}} \exp \left(-\frac{i \tau b}{2 a(\tau+a)}\right),
$$

with

$$
a=\frac{q_{0} x_{0}^{\prime}-p_{0} y_{0}^{\prime}}{q_{0} p_{0}^{\prime}-p_{0} q_{0}^{\prime}} \quad \text { and } \quad b=\left(\frac{v_{0}^{\prime}}{q_{0} p_{0}^{\prime}-p_{0} q_{0}^{\prime}}\right)^{2},
$$

and $A_{0}(s, 0)$ (which is either known or calculable) being the amplitude along the boundary. Higherorder corrections can be determined by solving the recursive relation given by the transport equation (2.5). This now completes solution to the leading-order Friedlander-Keller ray expansion.

The three classes of problems considered here are reflection from and transmission through an irregular boundary of the profile given by boundary (1.2) which separates two wave-bearing media supporting either $(i)$ acoustic waves, (ii) electromagnetic waves, or (iii) a single incident wave giving rise to two types of reflected wave as is the case with an incident wave in a semi-infinite elastic solid. In any case, the aim is to determine the contribution that this class of boundary makes to the reflected and transmitted fields.

The method to determine the required solutions will involve balancing, firstly, the $O(k)$ terms, and secondly the $O\left(k^{1 / 2}\right)$ terms which arise in the various exponents, as well as, thirdly, the terms which appear at $O(1)$ in the amplitude pre-factor to the exponential phase. This is achieved by taking a Taylor series expansion of the phase functions (for any wave) about the unperturbed boundary, giving

$$
\begin{aligned}
{\left[i k u+i k^{1 / 2} v\right]_{\partial \hat{D}} \sim i k u_{0}+i k^{1 / 2}\left[v_{0}+\boldsymbol{p}_{0} \cdot \hat{\boldsymbol{x}}_{0}\right] } \\
+\frac{i\left(q_{0} \hat{x}_{0}-p_{0} \hat{y}_{0}\right)}{2 p_{0} \boldsymbol{n} \cdot \boldsymbol{p}_{0}}\left(2 p_{0} v_{0}^{\prime}-\left(q_{0} \hat{x}_{0}-p_{0} \hat{y}_{0}\right) q_{0}^{\prime}\right), \\
{\left[i \frac{k}{\gamma} u+i\left(\frac{k}{\gamma}\right)^{1 / 2} v\right]_{\partial \hat{D}} \sim i k \frac{u_{0}}{\gamma}+i k^{1 / 2} \frac{\gamma^{1 / 2} v_{0}+\boldsymbol{p}_{0} \cdot \hat{\boldsymbol{x}}_{0}}{\gamma} } \\
+\frac{i\left(q_{0} \hat{x}_{0}-p_{0} \hat{y}_{0}\right)}{2 \gamma p_{0} \boldsymbol{n} \cdot \boldsymbol{p}_{0}}\left(2 \gamma^{1 / 2} p_{0} v_{0}^{\prime}-\left(q_{0} \hat{x}_{0}-p_{0} \hat{y}_{0}\right) q_{0}^{\prime}\right),
\end{aligned}
$$

where a zero subscript denotes the value of that quantity when evaluated along the unperturbed boundary $\partial D$. Note that, for example, the terms appearing at $O\left(k^{1 / 2}\right)$ in expansion (2.15) - expansion (2.16) contain two terms, one of which is the second term Taylor series expansion of $u$ and the other term is the leading-order term from the Taylor series expansion for $v$. The $O(1)$ terms in expansion (2.15) expansion (2.16) form part of the boundary data for the leading-order amplitude term $A_{0}$. 


\section{Reflection and transmission of acoustic waves at a perturbed boundary of general curvature}

\subsection{The boundary value problem for acoustic waves}

The total potential within the domain $\hat{D}, \phi^{T}$, is given by the superposition of the incident field, $\phi^{(i n c)}$, and the reflected field, $\phi^{r}$, and satisfies the Helmholtz equation (1.1)

$$
\frac{\partial^{2} \phi^{T}}{\partial x^{2}}+\frac{\partial^{2} \phi^{T}}{\partial y^{2}}+k^{2} \phi^{T}=0 .
$$

The total potential in the remaining region, $\phi^{t}$, is given solely by the transmitted disturbance and satisfies the second Helmholtz equation (1.3)

$$
\frac{\partial^{2} \phi^{t}}{\partial x^{2}}+\frac{\partial^{2} \phi^{t}}{\partial y^{2}}+\frac{k^{2}}{\gamma^{2}} \phi^{t}=0
$$

with $\gamma$ defining the ratio of the speed of sound within the two regions. The aim is to determine the leading-order solution for both $\phi^{r}$ and $\phi^{t}$ by solving both Helmholtz equations (3.1) and (3.2), subject to the coupled boundary data

$$
\begin{aligned}
{\left[\frac{\partial \phi^{t}}{\partial \hat{n}}\right]_{\partial \hat{D}} } & =\alpha\left[\frac{\partial \phi^{T}}{\partial \hat{n}}\right]_{\partial \hat{D}}, \\
{\left[\phi^{t}\right]_{\partial \hat{D}} } & =\beta\left[\phi^{T}\right]_{\partial \hat{D}}
\end{aligned}
$$

Here, $\alpha$ and $\beta$ are both $O(1)$ constants and are left general to maximise applicability; taking $\alpha=1$ and $\beta$ to be a ratio of fluid densities would model the interfacial conditions valid between two compressible fluids.

\subsection{Plane wave incidence}

Guided by all of the above, the leading-order solution for the reflected and transmitted fields are written in the form

$$
\begin{aligned}
\phi^{T} & \sim F(x \tan (\theta)+y) \exp (i k[x \cos (\theta)-y \sin (\theta)])+A_{0}^{r} \exp \left(i k u^{r}+i k^{1 / 2} v^{r}\right) \\
\phi^{t} & \sim A_{0}^{t} \exp \left(i \frac{k}{\gamma} u^{t}+i\left(\frac{k}{\gamma}\right)^{1 / 2} v^{t}\right)
\end{aligned}
$$

in which $A_{0}^{r}$ and $A_{0}^{t}$ are the leading-order amplitudes for the reflected and transmitted fields respectively. The potentials $\phi^{T}$ and $\phi^{t}$ satisfy the coupled boundary conditions (3.3) - (3.4), where all phase terms must be balanced (using either expansion (2.15) or expansion (2.16) where appropriate). At $O(k)$, these expansions provides the two boundary values for $u^{r}$ and $u^{t}$. Likewise, at $O\left(k^{1 / 2}\right)$, these expansions provide the boundary data for $v^{r}$ and $v^{t}$. Hence, the problem for $u^{r}$ and $u^{t}$ is to solve the eikonal equation (2.2) for each of these two functions subject to the boundary data

$$
u_{0}^{r}=x_{0} \cos (\theta)-y_{0} \sin (\theta) \quad \text { and } \quad u_{0}^{t}=\gamma\left[x_{0} \cos (\theta)-y_{0} \sin (\theta)\right],
$$

so that the full solutions for $u^{r}$ and $u^{t}$ are

$u^{r}\left(s, \tau^{r}\right)=x_{0} \cos (\theta)-y_{0} \sin (\theta)+\tau^{r} \quad$ and $\quad u^{t}\left(s, \tau^{t}\right)=\gamma\left[x_{0} \cos (\theta)-y_{0} \sin (\theta)\right]+\tau^{t},(3.8$ 
with $\tau^{r}$ and $\tau^{t}$ being the arc-lengths of the reflected and transmitted rays respectively. With $u^{r}$ and $u^{t}$ now known, the ray directions can be fixed from identity (2.10) with respect to the radiation condition assuring that the reflected and transmitted rays are emitted way from $\partial \hat{D}$ into and out of $\hat{D}$ respectively, the directions being

$$
\begin{aligned}
& p_{0}^{r}=\cos (\theta)\left(\left(x_{0}^{\prime}\right)^{2}-\left(y_{0}^{\prime}\right)^{2}\right)-2 \sin (\theta) x_{0}^{\prime} y_{0}^{\prime}, \\
& q_{0}^{r}=\sin (\theta)\left(\left(x_{0}^{\prime}\right)^{2}-\left(y_{0}^{\prime}\right)^{2}\right)+2 \cos (\theta) x_{0}^{\prime} y_{0}^{\prime}, \\
& p_{0}^{t}=\gamma x_{0}^{\prime}\left(\cos (\theta) x_{0}^{\prime}-\sin (\theta) y_{0}^{\prime}\right)+y_{0}^{\prime} \sqrt{1-\gamma^{2}\left(\cos (\theta) x_{0}^{\prime}-\sin (\theta) y_{0}^{\prime}\right)^{2}}, \\
& q_{0}^{t}=\gamma y_{0}^{\prime}\left(\cos (\theta) x_{0}^{\prime}-\sin (\theta) y_{0}^{\prime}\right)-x_{0}^{\prime} \sqrt{1-\gamma^{2}\left(\cos (\theta) x_{0}^{\prime}-\sin (\theta) y_{0}^{\prime}\right)^{2}} .
\end{aligned}
$$

These directions now provide the boundary data (and therefore the full solution) to $v^{r}$ and $v^{t}$, which are

$$
v^{r}(s)=-2\left(\boldsymbol{n} \cdot \boldsymbol{p}_{0}^{r}\right)\left(\boldsymbol{n} \cdot \hat{\boldsymbol{x}}_{0}\right) \quad \text { and } \quad v^{t}(s)=-\gamma^{-1 / 2}\left(\boldsymbol{n} \cdot \hat{\boldsymbol{x}}_{0}\right)\left(\boldsymbol{n} \cdot\left[\gamma \boldsymbol{p}_{0}^{r}+\boldsymbol{p}_{0}^{t}\right]\right),
$$

and now the remaining items to find are the two boundary values for the leading-order amplitudes. These are determined by simultaneously solving boundary condition (3.3) - boundary condition (3.4) (discarding the exponential terms at $O(k)$ and $O\left(k^{1 / 2}\right)$ as these have just been dealt with). Doing so gives that

$$
\begin{aligned}
A_{0}^{r}(s, 0)= & \frac{F\left(x_{0} \tan (\theta)+y_{0}\right)\left(\left[\alpha \gamma \boldsymbol{p}_{0}^{r}+\beta \boldsymbol{p}_{0}^{t}\right] \cdot \boldsymbol{n}\right)}{\left[\alpha \gamma \boldsymbol{p}_{0}^{r}-\beta \boldsymbol{p}_{0}^{t}\right] \cdot \boldsymbol{n}} \\
& \exp \left(2 i\left(q_{0}^{r} \hat{x}_{0}-p_{0}^{r} \hat{y}_{0}\right)\left(\boldsymbol{n} \cdot \hat{\boldsymbol{x}}_{0}^{\prime}\right)-i \frac{\left(q_{0}^{r} \hat{x}_{0}-p_{0}^{r} \hat{y}_{0}\right)^{2} \kappa_{0}}{\boldsymbol{n} \cdot \boldsymbol{p}_{0}^{r}}\right), \\
A_{0}^{t}(s, 0)= & \frac{2 \alpha \beta \gamma\left(\boldsymbol{n} \cdot \boldsymbol{p}_{0}^{r}\right) F\left(x_{0} \tan (\boldsymbol{\theta})+y_{0}\right)}{\left[\alpha \gamma \boldsymbol{p}_{0}^{r}-\beta \boldsymbol{p}_{0}^{t}\right] \cdot \boldsymbol{n}} \\
& \exp \left(\frac{i\left(q_{0}^{t} \hat{x}_{0}-p_{0}^{t} \hat{y}_{0}\right)\left(\left[\gamma \boldsymbol{p}_{0}^{r}+\boldsymbol{p}_{0}^{t}\right] \cdot \boldsymbol{n}\right)}{\gamma\left(\boldsymbol{n} \cdot \boldsymbol{p}_{0}^{t}\right)}\left[\left(\boldsymbol{n} \cdot \hat{\boldsymbol{x}}_{0}^{\prime}\right)-\frac{\kappa_{0}\left(q_{0}^{t} \hat{x}_{0}-p_{0}^{t} \hat{y}_{0}\right)}{2\left(\boldsymbol{n} \cdot \boldsymbol{p}_{0}^{t}\right)}\right]\right),
\end{aligned}
$$

where $\kappa_{0}$ is the curvature of the unperturbed boundary. The full solution for the two leading-order amplitudes of the reflected and transmitted fields are now given by solution (2.13) with the above boundary values and

$$
\begin{aligned}
a^{r}=-\frac{1}{2 \kappa_{0}} \boldsymbol{n} \cdot \boldsymbol{p}_{0}^{r} & \text { and } & b^{r}=\left(q_{0}^{r} \hat{x}_{0}-p_{0}^{r} \hat{y}_{0}-\frac{1}{\kappa_{0}}\left(\boldsymbol{n} \cdot \boldsymbol{p}_{0}^{r}\right)\left(\boldsymbol{n} \cdot \hat{\boldsymbol{x}}_{0}^{\prime}\right)\right)^{2} \\
a^{t}=-\frac{\left(\boldsymbol{n} \cdot \boldsymbol{p}_{0}^{t}\right)^{2}}{\kappa_{0}\left[\gamma \boldsymbol{p}_{0}^{r}+\boldsymbol{p}_{0}^{t}\right] \cdot \boldsymbol{n}} & \text { and } & b^{t}=\frac{1}{\gamma}\left(q_{0}^{t} \hat{x}_{0}-p_{0}^{t} \hat{y}_{0}-\frac{1}{\kappa_{0}}\left(\boldsymbol{n} \cdot \boldsymbol{p}_{0}^{t}\right)\left(\boldsymbol{n} \cdot \hat{\boldsymbol{x}}_{0}^{\prime}\right)\right)^{2}
\end{aligned}
$$

\subsection{Cylindrical wave incidence}

The total disturbance within $\hat{D}$ is given as

$$
\phi^{T} \sim \frac{F_{c}(\Theta)}{\sqrt{R}} \exp (i k R)+A_{0}^{r} \exp \left(i k u^{r}+i k^{1 / 2} v^{r}\right),
$$


with the first term of which specifies incident cylindrical wave and the second term representing the reflected field, and the transmitted field out of $\hat{D}$ is given by

$$
\phi^{t} \sim A_{0}^{t} \exp \left(i \frac{k}{\gamma} u^{t}+i\left(\frac{k}{\gamma}\right)^{1 / 2} v^{t}\right) .
$$

These two potentials $\phi^{T}$ and $\phi^{t}$ satisfy boundary conditions (3.3) and (3.4). The methodology is as before; the three phase terms appearing in the two potentials will be evaluated along $\partial \hat{D}$ by use of expansion (2.15) or expansion (2.16). Balancing the terms appearing at $O(k)$ give the boundary data for $u^{r}$ and $u^{t}$ as

$$
u_{0}^{r}=R_{0} \quad \text { and } \quad u_{0}^{t}=\gamma R_{0},
$$

in which $R_{0}=\sqrt{x_{0}^{2}+\left(y_{0}-h\right)^{2}}$. Thus, the general solutions for $u^{r}$ and $u^{t}$ are given as

$$
u^{r}\left(s, \tau^{r}\right)=R_{0}+\tau^{r} \quad \text { and } \quad u^{t}\left(s, \tau^{t}\right)=\gamma R_{0}+\tau^{r},
$$

with $\tau^{r}$ and $\tau^{t}$ being the arc-length of the reflected and transmitted ray respectively. The ray directions can then be determined complying with the radiation condition for both sets of rays, which give the directions

$$
\begin{aligned}
& p_{0}^{r}=\frac{1}{R_{0}}\left(2\left(y_{0}-h\right) x_{0}^{\prime} y_{0}^{\prime}+x_{0}\left(\left(x_{0}^{\prime}\right)^{2}-\left(y_{0}^{\prime}\right)^{2}\right)\right) \\
& q_{0}^{r}=\frac{1}{R_{0}}\left(2 x_{0} x_{0}^{\prime} y_{0}^{\prime}-\left(y_{0}-h\right)\left(\left(x_{0}^{\prime}\right)^{2}-\left(y_{0}^{\prime}\right)^{2}\right)\right)
\end{aligned}
$$

for the reflected waves, and

$$
\begin{aligned}
& p_{0}^{t}=\gamma R_{0}^{\prime} x_{0}^{\prime}+y_{0}^{\prime} \sqrt{1-\gamma^{2}\left(R_{0}^{\prime}\right)^{2}}, \\
& q_{0}^{t}=\gamma R_{0}^{\prime} y_{0}^{\prime}-x_{0}^{\prime} \sqrt{1-\gamma^{2}\left(R_{0}^{\prime}\right)^{2}},
\end{aligned}
$$

for those which are transmitted.

Comparison of the phase terms at $O\left(k^{1 / 2}\right)$ give the boundary data (and therefore the full solution) for $v^{r}$ and $v^{t}$, which are

$$
v^{r}=\frac{\boldsymbol{R}_{0} \cdot \hat{\boldsymbol{x}}_{0}}{R_{0}}-\hat{\boldsymbol{x}}_{0} \cdot \boldsymbol{p}_{0}^{r} \quad \text { and } \quad v^{t}=\frac{\sqrt{\gamma}\left(\boldsymbol{R}_{0} \cdot \hat{\boldsymbol{x}}_{0}\right)}{R_{0}}-\frac{\hat{\boldsymbol{x}}_{0} \cdot \boldsymbol{p}_{0}^{t}}{\sqrt{\gamma}}
$$

where $\boldsymbol{R}_{0}=\left(x_{0}, y_{0}-h\right)$.

Solving now the two boundary equations simultaneously whilst ignoring the exponential terms appearing at $O(k)$ and $O\left(k^{1 / 2}\right)$ (as these have just been dealt with) gives the leading-order amplitude of 
the reflected and transmitted waves along the boundary, which are

$$
\begin{aligned}
& A_{0}^{r}(s, 0)=\frac{F_{c}\left(\theta^{i}\right)\left(\left[\beta R_{0} \boldsymbol{p}_{0}^{t}-\alpha \gamma \boldsymbol{R}_{0}\right] \cdot \boldsymbol{n}\right)}{R_{0}^{3 / 2}\left(\left[\alpha \gamma \boldsymbol{p}_{0}^{r}-\beta \boldsymbol{p}_{0}^{t}\right] \cdot \boldsymbol{n}\right)} \\
& \exp \left(\frac{i\left(x_{0} \hat{y}_{0}-\left(y_{0}-h\right) \hat{x}_{0}\right)^{2}}{2 R_{0}^{3}}+\frac{i\left(q_{0}^{r} \hat{x}_{0}-p_{0}^{r} \hat{y}_{0}\right)}{\boldsymbol{n} \cdot \boldsymbol{p}_{0}^{r}}\left[\frac{\left(\hat{\boldsymbol{x}}_{\mathbf{0}} \cdot \boldsymbol{R}_{0}\right)\left(\boldsymbol{t} \cdot \boldsymbol{p}_{0}^{r}\right)}{R_{0}^{2}}\right.\right. \\
& \left.\left.-\frac{1}{R_{0}} \frac{d}{d s}\left[\boldsymbol{R}_{0} \cdot \hat{\boldsymbol{x}}_{0}\right]+\hat{\boldsymbol{x}}_{0}^{\prime} \cdot \boldsymbol{p}_{0}^{r}-\frac{\left(q_{0}^{r} \hat{x}_{0}-p_{0}^{r} \hat{y}_{0}\right) \kappa_{0}}{2}+\frac{q_{0}^{r} \hat{x}_{0}-p_{0}^{r} \hat{y}_{0}}{2\left(\boldsymbol{n} \cdot \boldsymbol{p}_{0}^{r}\right)} R_{0}^{\prime \prime}\right]\right), \\
& A_{0}^{t}(s, 0)=\frac{\alpha \beta \gamma F_{c}\left(\theta^{i}\right)\left(\left[R_{0} \boldsymbol{p}_{0}^{r}-\boldsymbol{R}_{0}\right] \cdot \boldsymbol{n}\right)}{R_{0}^{3 / 2}\left(\left[\alpha \gamma \boldsymbol{p}_{0}^{r}-\beta \boldsymbol{p}_{0}^{t}\right] \cdot \boldsymbol{n}\right)} \\
& \exp \left(\frac{i\left(x_{0} \hat{y}_{0}-\left(y_{0}-h\right) \hat{x}_{0}\right)^{2}}{2 R_{0}^{3}}+\frac{i\left(q_{0}^{t} \hat{x}_{0}-p_{0}^{t} \hat{y}_{0}\right)}{\boldsymbol{n} \cdot \boldsymbol{p}_{0}^{t}}\left[\frac{\left(\hat{\boldsymbol{x}}_{\mathbf{0}} \cdot \boldsymbol{R}_{0}\right)\left(\boldsymbol{t} \cdot \boldsymbol{p}_{0}^{t}\right)}{\gamma R_{0}^{2}}\right.\right. \\
& -\frac{1}{R_{0}} \frac{d}{d s}\left[\boldsymbol{R}_{0} \cdot \hat{\boldsymbol{x}}_{0}\right]+\frac{1}{\gamma}\left(\hat{\boldsymbol{x}}_{0}^{\prime} \cdot \boldsymbol{p}_{0}^{t}\right)-\frac{\left(q_{0}^{t} \hat{x}_{0}-p_{0}^{t} \hat{y}_{0}\right) \kappa_{0}}{2 \gamma} \\
& \left.\left.+\frac{q_{0}^{t} \hat{x}_{0}-p_{0}^{t} \hat{y}_{0}}{2\left(\boldsymbol{n} \cdot \boldsymbol{p}_{0}^{t}\right)} R_{0}^{\prime \prime}\right]\right) \text {. }
\end{aligned}
$$

The full solution for the two leading-order amplitudes of the reflected and transmitted waves are now given by solution (2.13) with the above boundary values and, with $\kappa_{0}$ denoting the curvature of the unperturbed boundary:

$$
\begin{aligned}
a^{r} & =-\frac{\left(\boldsymbol{n} \cdot \boldsymbol{p}_{0}^{r}\right)^{2}}{\kappa_{0}\left(\boldsymbol{n} \cdot \boldsymbol{p}_{0}^{r}\right)-R_{0}^{\prime \prime}}, \\
b^{r} & =\left(q_{0}^{r} \hat{x}_{0}-p_{0}^{r} \hat{y}_{0}-\frac{\boldsymbol{n} \cdot \boldsymbol{p}_{0}^{r}}{\kappa_{0}\left(\boldsymbol{n} \cdot \boldsymbol{p}_{0}^{r}\right)-R_{0}^{\prime \prime}}\left(\boldsymbol{p}_{0}^{r} \cdot \hat{\boldsymbol{x}}_{0}^{\prime}-\frac{1}{R_{0}} \frac{d}{d s}\left[\boldsymbol{R}_{0} \cdot \hat{\boldsymbol{x}}_{0}\right]+\frac{\left(\boldsymbol{R}_{0} \cdot \hat{\boldsymbol{x}}_{0}\right)\left(\boldsymbol{t} \cdot \boldsymbol{p}_{0}^{r}\right)}{R_{0}^{2}}\right)\right)^{2}, \\
a^{t}= & -\frac{\left(\boldsymbol{n} \cdot \boldsymbol{p}_{0}^{t}\right)^{2}}{\kappa_{0}\left(\boldsymbol{n} \cdot \boldsymbol{p}_{0}^{t}\right)-\gamma R_{0}^{\prime \prime}}, \\
b^{t}= & \frac{1}{\gamma}\left(\frac{\boldsymbol{n} \cdot \boldsymbol{p}_{0}^{t}}{\kappa_{0}\left(\boldsymbol{n} \cdot \boldsymbol{p}_{0}^{t}\right)-\gamma R_{0}^{\prime \prime}}\right)^{2}\left(\left(\boldsymbol{p}_{0}^{t} \cdot \hat{\boldsymbol{x}}_{0}^{\prime}\right)-\left(q_{0}^{t} \hat{x}_{0}-p_{0}^{t} \hat{y}_{0}\right) \kappa_{0}-\frac{\gamma}{\left.R_{0} \cdot \boldsymbol{p}_{0}^{t}\right)} \frac{d}{R_{0}^{2}}\left[\frac{\gamma\left(q_{0}^{t} \hat{x}_{0}-p_{0}^{t} \hat{y}_{0}\right)}{\boldsymbol{n} \cdot \boldsymbol{x}_{0}^{t}} R_{0}^{\prime \prime}\right)^{2},\right.
\end{aligned}
$$

and both solutions are now complete to leading-order. 


\section{Reflection and transmission of electromagnetic waves at a perturbed boundary of general cur- vature}

\subsection{The boundary value problem for electromagnetic waves}

In an isotropic, homogeneous, and charge-free vacuum, Maxwell's equations for the electric $\boldsymbol{E}(\boldsymbol{x}) e^{-i \omega t}$ and magnetic $\boldsymbol{B}(\boldsymbol{x}) e^{-i \omega t}$ fields are

$$
\begin{array}{rll}
\boldsymbol{\nabla} \times \boldsymbol{E}=i \omega \boldsymbol{B} & \text { and } & \boldsymbol{\nabla} \times \boldsymbol{B}=-\frac{i \omega}{c^{2}} \boldsymbol{E}, \\
\boldsymbol{\nabla} \cdot \boldsymbol{E}=0 & \text { and } & \boldsymbol{\nabla} \cdot \boldsymbol{B}=0,
\end{array}
$$

with $c$ being the speed of light. Strictly speaking, the divergence-free conditions (4.2) are both immediate consequences of the partial differential equations (4.1) in this time-harmonic context, but they can be used to show that $\boldsymbol{E}$ satisfies the vector Helmholtz equation

$$
\frac{\partial^{2} \boldsymbol{E}}{\partial x^{2}}+\frac{\partial^{2} \boldsymbol{E}}{\partial y^{2}}+k^{2} \boldsymbol{E}=\mathbf{0}
$$

where $k=\frac{\omega}{c}$ and that $\boldsymbol{B}$ satisfies the same vectorial Helmholtz equation and is related to $\boldsymbol{E}$ by

$$
\boldsymbol{B}=-\frac{i}{c k} \boldsymbol{\nabla} \times \boldsymbol{E} .
$$

It now follows that each component of $\boldsymbol{E}$ and $\boldsymbol{B}$ must now satisfy their own scalar Helmholtz equation (1.1), and so applying a Friedlander-Keller expansion of the form given by ansatz (2.1) to each of these components allows the theory leading to solution (2.13) to be applied. It follows that the exponents and square-root amplitude terms appearing in the solution to each component would necessarily be identical, allowing the components to be recombined and the leading-order vectorial amplitude to be written directly as

$$
\boldsymbol{E}_{0}(s, \tau)=\boldsymbol{E}_{0}(s, 0) \sqrt{\frac{a}{\tau+a}} \exp \left(-\frac{i \tau b}{2 a(\tau+a)}\right)
$$

with $a$ and $b$ defined by those formulae in (2.14), and with an exactly similar result holding for $\boldsymbol{B}_{0}$. These results can also be obtained directly by assuming Friedlander-Keller ray expansions for $\boldsymbol{E}$ and $\boldsymbol{B}$ in a vectorial form of the ansatz (2.1) with $A_{n}$ replaced by $\boldsymbol{E}_{n}$ and $\boldsymbol{B}_{n}$ respectively, and substituting them into Maxwell's equations (4.1) and (4.2). It is important to note that this procedure will only work if the "phase exponents" $u$ and $v$ are the same for both $\boldsymbol{E}$ and $\boldsymbol{B}$. Hence, the algebraic and exponential factors - and the functions contained within them - appearing in the right-hand side of the solution (4.5) will also be the same in both cases.

In fact, this procedure very quickly reveals that, at leading-order, Maxwell equation (4.1) yields

$$
\boldsymbol{B}_{0}=\frac{1}{c}\left(\nabla u \times \boldsymbol{E}_{0}\right) \quad \text { and } \quad \boldsymbol{E}_{0}=c\left(\boldsymbol{B}_{0} \times \nabla u\right) .
$$

Eliminating either $\boldsymbol{E}_{0}$ or $\boldsymbol{B}_{0}$ quickly confirms the eikonal equation (2.2) for $u$. Also notice that once $u$ (and hence $\boldsymbol{\nabla} u$ ) and $\boldsymbol{E}_{0}$ have been computed, then $\boldsymbol{B}_{0}$ follows immediately from the first of the equations 
in (4.6). It also follows that $\boldsymbol{E}_{0}, \boldsymbol{B}_{0}$, and $\boldsymbol{\nabla} u$ (the direction of propagation along the rays) form a mutually orthogonal set establishing (at leading-order) the transverse nature of these electromagnetic wave fields. Given this, and the fact that attention has been restricted to incident electric, $\boldsymbol{E}$, fields - the case of an incident magnetic, $\boldsymbol{B}$, field is procedurally identical - allowing the determination of $\boldsymbol{B}_{0}$ to be ignored henceforth, as it is a consequence of $\boldsymbol{E}_{0}$.

In fact, since equation (4.6) implies that $\boldsymbol{\nabla} u \cdot \boldsymbol{E}_{0}=0$, and since $\boldsymbol{\nabla} u=\boldsymbol{p}_{0}=\left(p_{0}, q_{0}\right)$, in this twodimensional case, the calculation of two components of $\boldsymbol{E}_{0}$ can be reduced to the single scalar amplitude $E_{0}(s, \tau)$ given by

$$
\boldsymbol{E}_{0}(s, \tau)=E_{0}(s, \tau)\left(q_{0}(s),-p_{0}(s)\right)
$$

once $p_{0}$ and $q_{0}$ are known. Equation (4.7) yields that $\boldsymbol{E}_{0}(s, 0)=E_{0}(s, 0)\left(q_{0}(s),-p_{0}(s)\right)$, and the plan is to calculate each $E_{0}(s, 0)$ appropriate for the reflected and transmitted electric fields, given the equivalent for the incident field, via the two boundary conditions

$$
\begin{aligned}
{\left[\hat{\boldsymbol{t}} \cdot \boldsymbol{E}^{(i)}\right]_{i=1}^{i=2} } & =0, \\
{\left[\varepsilon_{i} \hat{\boldsymbol{n}} \cdot \boldsymbol{E}^{(i)}\right]_{i=1}^{i=2} } & =0,
\end{aligned}
$$

in which $[A]_{i=1}^{i=2}=0$ implies continuity of the quantity $A$ across the perturbed boundary $\partial \hat{D}, \boldsymbol{E}^{(i)}$ is the total electric field, and $\varepsilon$ is the electric permittivity on either side of that boundary, with $\hat{\boldsymbol{t}}$ and $\hat{\boldsymbol{n}}$ being the local boundary tangential and normal vectors. These boundary conditions can be simplified to leading-order by noting that, in this two-dimensional case, $\hat{\boldsymbol{t}} \sim \boldsymbol{t}=\left(x_{0}^{\prime}, y_{0}^{\prime}\right), \hat{\boldsymbol{n}} \sim \boldsymbol{n}=\left(-y_{0}^{\prime}, x_{0}^{\prime}\right), \boldsymbol{E}^{(i)} \sim$ $\boldsymbol{E}_{0}^{(i n c)}+\boldsymbol{E}_{0}^{r}$, and $\boldsymbol{E}^{(2)} \sim \boldsymbol{E}_{0}^{t}$, where the superscripts "(inc)", " $r$ ", and " $t$ " refer to incident, reflected, and transmitted electric fields, respectively. Also notice that the $\boldsymbol{E}_{0}^{t}$ field propagated in a vacuum of refractive index $\gamma^{-1}$, so that $k$ is replaced by $\frac{k}{\gamma}$ in the relevant Friedlander - Keller ray expansions.

\subsection{Plane wave incidence}

In the case of an incident plane electromagnetic wave, the leading-order electric fields are, in the previous notation

$$
\begin{aligned}
\boldsymbol{E}^{(1)} \sim & E_{0}^{r}\left(q_{0}^{r},-p_{0}^{r}\right) \exp \left(i k u^{r}+i k^{1 / 2} v^{r}\right) \\
& -F_{p}(x \tan (\theta)+y)(\sin (\theta), \cos (\theta)) \exp (i k[x \cos (\theta)-y \sin (\theta)]), \\
\boldsymbol{E}^{(2)} \sim & E_{0}^{t}\left(q_{0}^{t},-p_{0}^{t}\right) \exp \left(i \frac{k}{\gamma} u^{t}+i\left(\frac{k}{\gamma}\right)^{1 / 2} v^{t}\right)
\end{aligned}
$$

where the arbitrary function $F_{p}$ determines the amplitude profile of the incident wave. The exponents which appear in these two fields are exactly similar to those appearing in the case of an incident plane acoustic wave, and so it follows that the four phase functions and the ray directions are given by solutions (3.7) - (3.13) inclusive. The boundary amplitudes $\boldsymbol{E}_{0}^{r}$ and $\boldsymbol{E}_{0}^{t}$ (so that, for example, $\left.\boldsymbol{E}_{0}^{r}(s, 0)=E_{0}^{r}(s, 0)\left(q_{0}^{r}(s),-p_{0}^{r}(s)\right)\right)$ are now calculated by simultaneously solving boundary conditions 
(4.8) and (4.9), giving

$$
\begin{aligned}
\boldsymbol{E}_{0}^{r}(s, 0)= & \frac{F_{p}\left(x_{0} \tan (\boldsymbol{\theta})+y_{0}\right)\left[\varepsilon_{1}\left(\boldsymbol{t} \cdot \boldsymbol{p}_{0}^{r}\right)\left(\boldsymbol{n} \cdot \boldsymbol{p}_{0}^{t}\right)+\varepsilon_{2}\left(\boldsymbol{t} \cdot \boldsymbol{p}_{0}^{t}\right)\left(\boldsymbol{n} \cdot \boldsymbol{p}_{0}^{r}\right)\right]}{\left(\left[\gamma \varepsilon_{2} \boldsymbol{p}_{0}^{r}-\varepsilon_{1} \boldsymbol{p}_{0}^{t}\right] \cdot \boldsymbol{n}\right)\left(\boldsymbol{t} \cdot \boldsymbol{p}_{0}^{r}\right)}\left(q_{0}^{r},-p_{0}^{r}\right) \\
& \exp \left(2 i\left(q_{0}^{r} \hat{x}_{0}-p_{0}^{r} \hat{y}_{0}\right)\left(\boldsymbol{n} \cdot \hat{\boldsymbol{x}}_{0}^{\prime}\right)-\frac{i\left(q_{0}^{r} \hat{x}_{0}-p_{0}^{r} \hat{y}_{0}\right)^{2} \kappa_{0}}{\boldsymbol{n} \cdot \boldsymbol{p}_{0}^{r}}\right), \\
\boldsymbol{E}_{0}^{t}(s, 0)= & \frac{2 \varepsilon_{1} F_{p}\left(x_{0} \tan (\theta)+y_{0}\right)\left(\boldsymbol{n} \cdot \boldsymbol{p}_{0}^{r}\right)}{\left[\gamma \varepsilon_{2} \boldsymbol{p}_{0}^{r}-\varepsilon_{1} \boldsymbol{p}_{0}^{t}\right] \cdot \boldsymbol{n}}\left(q_{0}^{t},-p_{0}^{t}\right) \\
& \exp \left(\frac{i\left(q_{0}^{t} \hat{x}_{0}-p_{0}^{t} \hat{y}_{0}\right)\left(\left[\gamma \boldsymbol{p}_{0}^{r}+\boldsymbol{p}_{0}^{t}\right] \cdot \boldsymbol{n}\right)}{\gamma\left(\boldsymbol{n} \cdot \boldsymbol{p}_{0}^{t}\right)}\left[\boldsymbol{n} \cdot \hat{\boldsymbol{x}}_{0}^{\prime}-\frac{\kappa_{0}\left(q_{0}^{t} \hat{x}_{0}-p_{0}^{t} \hat{y}_{0}\right)}{2\left(\boldsymbol{n} \cdot \boldsymbol{p}_{0}^{t}\right)}\right]\right) .
\end{aligned}
$$

The leading-order solutions for the reflected and transmitted fields are now given by the solution (4.5) with the $a$ and $b$ functions defined as in solutions (3.16) and (3.17) respectively.

\subsection{Cylindrical wave incidence}

Following an identical methodology, the leading-order total and transmitted fields are contained within

$$
\begin{aligned}
& \boldsymbol{E}^{(1)} \sim E_{0}^{r}\left(q_{0}^{r},-p_{0}^{r}\right) \exp \left(i k u^{r}+i k^{1 / 2} v^{r}\right)+\frac{F_{c}(\Theta)}{R^{3 / 2}}(y-h,-x) \exp (i k R) \\
& \boldsymbol{E}^{(2)} \sim E_{0}^{t}\left(q_{0}^{t},-p_{0}^{t}\right) \exp \left(i \frac{k}{\gamma} u^{t}+i\left(\frac{k}{\gamma}\right)^{1 / 2} v^{t}\right)
\end{aligned}
$$

where $F_{c}(\Theta)$ is the angular directivity of the cylindrically-spreading incident field emanating from the source point $(0, h)$. Again, the exponents which appear in these two fields are exactly similar to those appearing in the case of an incident acoustic cylindrical wave, and so it follows that the four phase functions and the ray directions are given by the formulae appearing in (3.20) - (3.26).

The boundary amplitudes $\boldsymbol{E}_{0}^{r}$ and $\boldsymbol{E}_{0}^{t}$ are now calculated in the same manner as in the previous 
subsection, giving that

$$
\begin{aligned}
\boldsymbol{E}_{0}^{r}(s, 0)= & \frac{F_{c}(\Theta)\left[\varepsilon_{1}\left(\boldsymbol{t} \cdot \boldsymbol{R}_{0}\right)\left(\boldsymbol{n} \cdot \boldsymbol{p}_{0}^{t}\right)-\varepsilon_{2}\left(\boldsymbol{t} \cdot \boldsymbol{p}_{0}^{t}\right)\left(\boldsymbol{n} \cdot \boldsymbol{R}_{0}\right)\right]}{R_{0}^{3 / 2}\left(\left[\gamma \varepsilon_{2} \boldsymbol{p}_{0}^{r}-\varepsilon_{1} \boldsymbol{p}_{0}^{t}\right] \cdot \boldsymbol{n}\right)\left(\boldsymbol{t} \cdot \boldsymbol{p}_{0}^{r}\right)}\left(q_{0}^{r},-p_{0}^{r}\right) \\
& \exp \left(\frac{i\left(x_{0} \hat{y}_{0}-\left(y_{0}-h\right) \hat{x}_{0}\right)^{2}}{2 R_{0}^{3}}+\frac{i\left(q_{0}^{r} \hat{x}_{0}-p_{0}^{r} \hat{y}_{0}\right)}{\boldsymbol{n} \cdot \boldsymbol{p}_{0}^{r}}\left[\frac{\left(\hat{\boldsymbol{x}}_{\mathbf{0}} \cdot \boldsymbol{R}_{0}\right)\left(\boldsymbol{t} \cdot \boldsymbol{p}_{0}^{r}\right)}{R_{0}^{2}}\right.\right. \\
& \left.\left.-\frac{1}{R_{0}} \frac{d}{d s}\left[\boldsymbol{R}_{0} \cdot \hat{\boldsymbol{x}}_{0}\right]+\hat{\boldsymbol{x}}_{0}^{\prime} \cdot \boldsymbol{p}_{0}^{r}-\frac{\left(q_{0}^{r} \hat{x}_{0}-p_{0}^{r} \hat{y}_{0}\right) \kappa_{0}}{2}+\frac{q_{0}^{r} \hat{x}_{0}-p_{0}^{r} \hat{y}_{0}}{2\left(\boldsymbol{n} \cdot \boldsymbol{p}_{0}^{r}\right)} \boldsymbol{R}_{0}^{\prime \prime}\right]\right) \\
\boldsymbol{E}_{0}^{t}(s, 0)= & \frac{\varepsilon_{1} F_{c}(\Theta)\left[q_{0}^{r} x_{0}-p_{0}^{r}\left(y_{0}-h\right)\right]}{R_{0}^{3 / 2}\left(\left[\gamma \varepsilon_{2} \boldsymbol{p}_{0}^{r}-\varepsilon_{1} \boldsymbol{p}_{0}^{t}\right] \cdot \boldsymbol{n}\right)\left(\boldsymbol{t} \cdot \boldsymbol{p}_{0}^{r}\right)}\left(q_{0}^{t},-p_{0}^{t}\right) \\
& \quad \exp \left(\frac{i\left(x_{0} \hat{y}_{0}-\left(y_{0}-h\right) \hat{x}_{0}\right)^{2}}{2 R_{0}^{3}}+\frac{i\left(q_{0}^{t} \hat{x}_{0}-p_{0}^{t} \hat{y}_{0}\right)}{\boldsymbol{n} \cdot \boldsymbol{p}_{0}^{t}}\left[\frac{\left(\hat{\boldsymbol{x}}_{\mathbf{0}} \cdot \boldsymbol{R}_{0}\right)\left(\boldsymbol{t} \cdot \boldsymbol{p}_{0}^{t}\right)}{\gamma R_{0}^{2}}\right.\right. \\
& \left.\left.-\frac{1}{R_{0}} \frac{d}{d s}\left[\boldsymbol{R}_{0} \cdot \hat{\boldsymbol{x}}_{0}\right]+\frac{1}{\gamma} \hat{\boldsymbol{x}}_{0}^{\prime} \cdot \boldsymbol{p}_{0}^{t}-\frac{\left(q_{0}^{t} \hat{x}_{0}-p_{0}^{t} \hat{y}_{0}\right) \kappa_{0}}{2 \gamma}+\frac{q_{0}^{t} \hat{x}_{0}-p_{0}^{t} \hat{y}_{0}}{2\left(\boldsymbol{n} \cdot \boldsymbol{p}_{0}^{t}\right)} R_{0}^{\prime \prime}\right]\right) .
\end{aligned}
$$

The leading-order solutions for the reflected and transmitted fields are now given by the solution (4.5) with the $a$ and $b$ functions defined as those in solutions (3.29) - (3.32).

\section{Reflection and transmission of elastic waves at a perturbed boundary of general curvature}

\subsection{The boundary value problem for elastic waves}

The elastic displacement vector, $\boldsymbol{u}(\boldsymbol{x}) e^{-i \omega t}$, can, via the Helmholtz decomposition, always be written in the form

$$
\boldsymbol{u}(\boldsymbol{x})=\boldsymbol{\nabla} \phi(\boldsymbol{x})+(\boldsymbol{\nabla} \times \boldsymbol{\chi}(\boldsymbol{x})) ; \boldsymbol{\chi}(\boldsymbol{x})=0
$$

and in the situation of plane strain that is considered here, $\phi$ and $\chi$ can be taken to be $\phi=\phi(x, y)$, and $\boldsymbol{\chi}=\chi(x, y) \boldsymbol{e}_{z}$ (so that $\boldsymbol{\nabla} \cdot \boldsymbol{\chi}=0$ is automatically satisfied). The $\boldsymbol{\nabla} \boldsymbol{\phi}$ term in the first equation of (5.1) contributes a longitudinal disturbance to the displacement field $\boldsymbol{u}$, while $\chi$ yields a vertically polarised shear wave component. It is well known that all of these different wave modes can arise when an elastic field is incident upon a free surface, even if only one such field is incident (Brekhovskikh (1980), Graff (1991), and Howell et al (2008)). At such a free surface, where the traction vanishes, the boundary condition to be satisfied can (Gregory (1970)) be expressed in terms of $\phi$ and $\chi$ in the form

$$
2 \frac{\partial}{\partial \hat{n}}\left[\nabla \phi+\left(\nabla \chi \times \boldsymbol{e}_{z}\right)\right]+\left(2 k_{l}^{2}-k_{s}^{2}\right) \hat{\boldsymbol{n}} \phi+k_{s}^{2} \chi\left(\hat{\boldsymbol{n}} \times \boldsymbol{e}_{z}\right)=\mathbf{0}
$$

in which $k_{l}$ and $k_{s}$ are the wave-numbers of the reflected longitudinal and shear waves respectively.

The following examples contain only an incident longitudinal wave, and so the incident term is present in the $\phi$ field. In the case of an incident shear wave, the incident term would be present in the $\chi$ field, but the methodology would be the identical. 


\subsection{Longitudinal plane wave incidence}

Consider a plane wave propagated longitudinally which is incident upon a free surface, $\partial \hat{D}$. This gives rise to two reflected waves, one which is also longitudinal, and another which is a vertically polarised shear wave. Thus, since $\phi$ is the superposition of longitudinal waves, it is written as

$$
\begin{aligned}
\phi \sim & F_{p}(x \tan (\theta)+y) \exp (i k[x \cos (\theta)-y \sin (\theta)]) \\
& +A_{0}^{l} \exp \left(i k u^{l}+i k^{1 / 2} v^{l}\right),
\end{aligned}
$$

where $F_{p}$ specifies the amplitude profile of the incident longitudinal wave, and $\chi$ represents the reflected shear wave, and so has the leading-order form

$$
\chi \sim A_{0}^{s} \exp \left(i \frac{k}{\gamma} u^{s}+i\left(\frac{k}{\gamma}\right)^{1 / 2} v^{s}\right) .
$$

Comparing this situation to, say, the acoustic scattering considered previously, the "reflected" quantities there are the longitudinal quantities here, as are the "transmitted" and shear quantities. Balancing the exponents terms at $O(k)$ along the boundary $\partial \hat{D}$ gives the boundary data for $u^{l}$ and $u^{t}$, which are indifferent to the boundary values given in (3.7) if the " $r$ " and " $t$ " superscripts are replaced by "l" and " $s$ " respectively, and so the solution for these two functions is given as those appearing in solution (3.8), again with the change in superscripts, with $\tau^{l}$ and $\tau^{s}$ now denoting the arc-length of the reflected longitudinal and shear waves respectively. This also implies that the ray directions for the longitudinally reflected waves are the same as the ray directions appearing in (3.9) and (3.10), but the ray directions of the reflected shear waves differs from those in (3.11) and (3.12) as the sign of the second terms are changed to respect the decaying condition imposed on equation (2.10). This arises as both sets of rays are propagated back into the same medium (rather than only one reflecting back and then having one escape the medium as was the case for the acoustic scenario).

Furthermore, this also implies that both equations (3.13) still hold, again with the change in superscript. Simultaneously solving the boundary condition (5.2) for the leading-order amplitudes of the two sets of waves gives that

$$
\begin{aligned}
A_{0}^{l}(s, 0)= & \frac{F_{p}\left(x_{0} \tan (\boldsymbol{\theta})+y_{0}\right)\left[4 \gamma\left(\boldsymbol{n} \cdot \boldsymbol{p}_{0}^{l}\right)\left(\boldsymbol{n} \cdot \boldsymbol{p}_{0}^{s}\right)\left(\boldsymbol{t} \cdot \boldsymbol{p}_{0}^{s}\right)^{2}+\left[1+2\left(\boldsymbol{t} \cdot \boldsymbol{p}_{0}^{s}\right)^{2}\right]^{2}\right]}{4 \gamma\left(\boldsymbol{n} \cdot \boldsymbol{p}_{0}^{l}\right)\left(\boldsymbol{n} \cdot \boldsymbol{p}_{0}^{s}\right)\left(\boldsymbol{t} \cdot \boldsymbol{p}_{0}^{s}\right)^{2}+\left[1-2\left(\boldsymbol{t} \cdot \boldsymbol{p}_{0}^{s}\right)^{2}\right]^{2}} \\
& \exp \left(\frac{i\left(q_{0}^{l} \hat{x}_{0}-p_{0}^{l} \hat{y}_{0}\right)}{\boldsymbol{n} \cdot \boldsymbol{p}_{0}^{l}}\left[2\left(\boldsymbol{n} \cdot \boldsymbol{p}_{0}^{l}\right)\left(\boldsymbol{n} \cdot \hat{\boldsymbol{x}}_{0}^{\prime}\right)-\left(q_{0}^{l} \hat{x}_{0}-p_{0}^{l} \hat{y}_{0}\right) \kappa_{0}\right]\right), \\
A_{0}^{s}(s, 0)= & \frac{4 \gamma^{2} F_{p}\left(x_{0} \tan (\boldsymbol{\theta})+y_{0}\right)\left(\boldsymbol{t} \cdot \boldsymbol{p}_{0}^{l}\right)\left(\boldsymbol{n} \cdot \boldsymbol{p}_{0}^{l}\right)\left[1-2\left(\boldsymbol{t} \cdot \boldsymbol{p}_{0}^{s}\right)^{2}\right]}{4 \gamma\left(\boldsymbol{n} \cdot \boldsymbol{p}_{0}^{l}\right)\left(\boldsymbol{n} \cdot \boldsymbol{p}_{0}^{s}\right)\left(\boldsymbol{t} \cdot \boldsymbol{p}_{0}^{s}\right)^{2}+\left[1-2\left(\boldsymbol{t} \cdot \boldsymbol{p}_{0}^{s}\right)^{2}\right]^{2}} \\
& \exp \left(\frac { i ( q _ { 0 } ^ { s } \hat { x } _ { 0 } - p _ { 0 } ^ { s } \hat { y } _ { 0 } ) } { \boldsymbol { n } \cdot \boldsymbol { p } _ { 0 } ^ { s } } \left[\left(\frac{\left(\hat{\boldsymbol{x}}_{0} \cdot \boldsymbol{p}_{0}^{l}\right)\left(\boldsymbol{t} \cdot \boldsymbol{p}_{0}^{s}\right)}{2\left(\boldsymbol{n} \cdot \boldsymbol{p}_{0}^{s}\right)}-\frac{\boldsymbol{t} \cdot \hat{\boldsymbol{x}}_{0}}{2 \gamma\left(\boldsymbol{n} \cdot \boldsymbol{p}_{0}^{s}\right)}-\frac{q_{0}^{s} \hat{x}_{0}-p_{0}^{s} \hat{y}_{0}}{2}\right) \kappa_{0}\right.\right. \\
& \left.\left.+\frac{1}{\gamma}\left(\left[\gamma \boldsymbol{p}_{0}^{l}+\boldsymbol{p}_{0}^{s}\right] \cdot \boldsymbol{n}\right)\left(\boldsymbol{n} \cdot \hat{\boldsymbol{x}}_{0}^{\prime}\right)\right]\right),
\end{aligned}
$$


with the full field solution for $A_{0}^{l}$ and $A_{0}^{s}$ being given by the solution (2.13) with the $a$ and $b$ functions defined as those appearing in (3.16) and (3.17) (with the superscripts interchanged).

\subsection{Longitudinal cylindrical wave incidence}

Following the same methodology as before, consider an incident longitudinal wave spreading cylindrically from the Cartesian point $(0, h)$ which is incident upon a free surface and will give rise to both a longitudinal and shear reflected waves. In this case $\phi$ and $\chi$ have the leading order forms

$$
\begin{aligned}
& \phi \sim \frac{F_{c}(\Theta)}{\sqrt{R}} \exp (i k R)+A_{0}^{l} \exp \left(i k u^{l}+i k^{1 / 2} v^{l}\right), \\
& \chi \sim A_{0}^{s} \exp \left(i k \frac{u^{s}}{\gamma}+i k^{1 / 2} \frac{v^{s}}{\gamma^{1 / 2}}\right)
\end{aligned}
$$

where $F_{c}$ specifies the angular directivity of the cylindrically-spreading incident field. This situation can be compared to the case of an incident cylindrical acoustic wave, with the "reflected" quantities there becoming the longitudinal quantities here, as with the "transmitted" and the shear quantities. Balancing the exponential terms at $O(k)$ of the leading-order forms (5.7) and (5.8) along the boundary $\partial \hat{D}$ yield the boundary data for $u^{l}$ and $u^{s}$. It turns out that the boundary data for $u^{l}$ and $u^{s}$ are mathematically similar to boundary data (3.20) (simply replacing the superscripts " $r$ " with " $l$ " and " $t$ " with " $s$ " throughout). This means two things: Firstly, the solution for $u^{l}$ and $u^{s}$ are given by solution (3.21). Secondly, the ray directions for the longitudinally reflected waves are also given by equations (3.22) and (3.23). However, the ray directions for the shear reflected waves differ from those in equations (3.24) and (3.25) as the sign of the second term is negated. This negation is required to obey the radiation condition imposed on equation (2.10), which arises as both sets of rays are reflected back into the medium, rather than one set of rays leaving the medium.

Moreover, when evaluating the lower-order exponents terms of the leading-order solutions (5.7) and (5.8), these too are identical to those given by the solution (3.26) (again with the change of superscripts " $r$ " and " $t$ " with "l" and " $s ")$. Solving the boundary condition (5.2) for the two boundary amplitude 
values gives that

$$
\begin{aligned}
& A_{0}^{l}(s, 0)=\frac{F_{c}(\Theta)}{R_{0}^{5 / 2}} \times \frac{R_{0}^{2}\left[2\left(\boldsymbol{t} \cdot \boldsymbol{p}_{0}^{s}\right)-1\right]+2 \gamma^{2}\left(\boldsymbol{t} \cdot \boldsymbol{R}_{0}\right)\left[\left(\boldsymbol{t} \cdot \boldsymbol{R}_{0}\right)\left(1-2\left(\boldsymbol{t} \cdot \boldsymbol{p}_{0}^{s}\right)^{2}\right)-2\left(\boldsymbol{n} \cdot \boldsymbol{p}_{0}^{s}\right)\left(\boldsymbol{t} \cdot \boldsymbol{p}_{0}^{s}\right)\left(\boldsymbol{n} \cdot \boldsymbol{R}_{0}\right)\right]}{1+4\left(\boldsymbol{t} \cdot \boldsymbol{p}_{0}^{s}\right)^{2}\left[\left(\boldsymbol{t} \cdot \boldsymbol{p}_{0}^{s}\right)^{2}+\gamma\left(\boldsymbol{n} \cdot \boldsymbol{p}_{0}^{s}\right)\left(\boldsymbol{n} \cdot \boldsymbol{p}_{0}^{l}\right)-1\right]} \\
& \exp \left(\frac{i\left(x_{0} \hat{y}_{0}-\hat{x}_{0}\left(y_{0}-h\right)\right)^{2}}{2 R_{0}^{3}}+\frac{i\left(q_{0}^{l} \hat{x}_{0}-p_{0}^{l} \hat{y}_{0}\right)}{\boldsymbol{n} \cdot \boldsymbol{p}_{0}^{l}}\left[\frac{\left(\boldsymbol{t} \cdot \boldsymbol{p}_{0}^{l}\right)\left(\boldsymbol{R} \cdot \hat{\boldsymbol{x}}_{0}\right)}{R_{0}^{2}}+\boldsymbol{p}_{0}^{l} \cdot \hat{\boldsymbol{x}}_{0}^{\prime}\right.\right. \\
& \left.\left.-\frac{q_{0}^{l} \hat{x}_{0}-p_{0}^{l} \hat{y}_{0}}{2}-\frac{1}{R_{0}} \frac{d}{d s}\left[\boldsymbol{R}_{0} \cdot \hat{\boldsymbol{x}}_{0}\right]+\frac{q_{0}^{l} \hat{x}_{0}-p_{0}^{l} \hat{y}_{0}}{2\left(\boldsymbol{n} \cdot \boldsymbol{p}_{0}^{l}\right)} R_{0}^{\prime \prime}\right]\right), \\
& A_{0}^{s}(s, 0)=\frac{2 \gamma^{2} F_{c}(\Theta)}{R_{0}^{5 / 2}} \times \frac{\left(\boldsymbol{n} \cdot \boldsymbol{p}_{0}^{l}\right)\left(\boldsymbol{t} \cdot \boldsymbol{p}_{0}^{l}\right)\left[R_{0}^{2}-2 \gamma^{2}\left(\boldsymbol{t} \cdot \boldsymbol{R}_{0}\right)^{2}\right]-\left(\boldsymbol{n} \cdot \boldsymbol{R}_{0}\right)\left(\boldsymbol{t} \cdot \boldsymbol{R}_{0}\right)\left[1-2\left(\boldsymbol{t} \cdot \boldsymbol{p}_{0}^{s}\right)^{2}\right]}{R_{0}^{5 / 2}\left[1+4\left(\boldsymbol{t} \cdot \boldsymbol{p}_{0}^{s}\right)^{2}\left[\left(\boldsymbol{t} \cdot \boldsymbol{p}_{0}^{s}\right)^{2}+\gamma\left(\boldsymbol{n} \cdot \boldsymbol{p}_{0}^{s}\right)\left(\boldsymbol{n} \cdot \boldsymbol{p}_{0}^{l}\right)-1\right]\right]} \\
& \exp \left(\frac{i\left(x_{0} \hat{y}_{0}-\hat{x}_{0}\left(y_{0}-h\right)\right)^{2}}{2 R_{0}^{3}}+\frac{i\left(q_{0}^{s} \hat{x}_{0}-p_{0}^{s} \hat{y}_{0}\right)}{\boldsymbol{n} \cdot \boldsymbol{p}_{0}^{s}}\left[\frac{\left(\boldsymbol{t} \cdot \boldsymbol{p}_{0}^{s}\right)\left(\boldsymbol{R} \cdot \hat{\boldsymbol{x}}_{0}\right)}{\gamma R_{0}^{2}}+\frac{1}{\gamma}\left(\boldsymbol{p}_{0}^{s} \cdot \hat{\boldsymbol{x}}_{0}^{\prime}\right)\right.\right. \\
& \left.\left.-\frac{q_{0}^{l} \hat{x}_{0}-p_{0}^{l} \hat{y}_{0}}{2 \gamma}-\frac{1}{R_{0}} \frac{d}{d s}\left[\boldsymbol{R}_{0} \cdot \hat{\boldsymbol{x}}_{0}\right]+\frac{q_{0}^{s} \hat{x}_{0}-p_{0}^{s} \hat{y}_{0}}{2\left(\boldsymbol{n} \cdot \boldsymbol{p}_{0}^{s}\right)} R_{0}^{\prime \prime}\right]\right),
\end{aligned}
$$

from which the full field solution for $A_{0}^{l}$ and $A_{0}^{s}$ can be determined by solution (2.13) with the $a$ and $b$ functions defined as those appearing in (3.29) - (3.32), with the change of superscripts mentioned above.

\section{Discussion and concluding remarks}

The first comment regards the leading-order amplitude, which would be excited by the profile of an unperturbed boundary (occurring when $\tau=-a(s)$ in solution (2.13), for example). This remains the case if small-scale undulations of $O\left(k^{-1 / 2}\right)$ perturb the boundary. However, these perturbations introduce a phase correction which is given by the exponents in solution (2.13), and in which $\tau=-a(s)$ would now cause singularities in the phase.

It was stated at the outset of this paper that the case of $N=2$ (in boundary (1.2), for example) is a distinguished value, as this value was shown to be the only instance when there is precisely one additional term in the exponents (in addition to the term appearing at $O(k)$ ), and so a possible extension which isn't considered here is the application of scattering of acoustic, electromagnetic, and elastic waves for a different value of $N$. Tew (2018) considered the reflection of incident plane and incident cylindrical scalar waves against a boundary with $N=3$ in boundary (1.2) (on which Neumann data was imposed), and then found the three phase functions and the leading-order amplitude of the reflected field. The conclusion made there was that the case of $N=3$ sets a paradigm for larger values of $N$, and each value of $N$ must then be considered separately. However, as $F_{p}$ and $F_{c}$ (in the notation of expansions (1.4) and (1.5)) are expanded about the unperturbed boundary, and which have boundary data on $\tau=0$, these leading-order amplitudes are independent of the value of $N$. The $O(1)$ exponential pre-factor to these amplitudes will change, however, as these arise form the $O(1)$ terms in the Taylor series expansion of the $N$ phase functions in $\sum_{n=0}^{\infty} \frac{A_{n}(x, y)}{k^{n / N}} \exp \left(i \sum_{m=1}^{N} k^{(N+1-m)) / N} v_{m}(x, y)\right)$. This leads, naturally, to consider 
the relationship between a general case of $N=N_{0}$ and then the case of $N=N_{0}+1$.

Another extension to the work presented here (and which is currently under investigation) is the application of the Friedlander-Keller treatment to vectorial waves rather than to the scalar waves. This would involve the fully vectorial equations of Maxwell, rather than exploiting the "compartmentalisation" method which was used in Section 4, and would subsequently allow for the understanding of how the electric $\boldsymbol{E}$ and the magnetic $\boldsymbol{B}$ fields are affected by the presence of a two-dimensionally perturbed boundary, rather than considering a two-dimensional problem with a one-dimensional boundary. This would also allow for a full solution to the equations of Maxwell in the short wave-limit by considering the leading-order solution, and then arriving at a vectorial equivalent of the transport equations (2.5) which would involve decoupling entirely the $\boldsymbol{E}$ and $\boldsymbol{B}$ fields. The three-dimensional problem would involve a boundary of the form considered by boundary (1.2), but with two parameters (one for each principal direction of the two-dimensional boundary). This will be reported on in due course.

\section{Acknowledgement}

One of the authors (A.M.R. Radjen) is grateful to the University of Nottingham for the provision of a fully-funded Ph.D studentship during the course of this research.

\section{REFERENCES}

Brekhovskikh, L. M. (1980), Waves in Layered Media, London, Academic Press.

Engineer, J. C., King, J. R., \& TeW, R. H. (1998), Diffraction by slender bodies, European Journal of Applied Mathematics, 9, 129 - 158.

Friedlander, F. G. \& Keller, J. B. (1955), Asymptotic Expansions of Solutions of $\left(\nabla^{2}+k^{2}\right) u=0$, Communications of Pure and Applied Mathematics, 8, 387 - 394.

GrAFF, K. F. (1991), Wave Motion In Elastic Solids, New York, Dover.

GREGORY, R. D. (1970), The propagation of Rayleigh waves over curved surfaces at high frequency, Proceedings of the Cambridge Philosophical Society, 70, 103 - 121.

Howell, P, Kozyreff, G, \& Ockendon, J. (2008), Applied Solid Mechanics, Cambridge, Cambridge University Press.

Ockendon, J., Howison, S., Lacey, A., And Movchan, A., (2003) Applied Partial Differential Equations Revised Edition, U.S.A., Oxford University Press.

TEw, R. H. (2018), Asymptotic solutions of the Helmholtz equation: Friendlander-Keller ray expansions of fractional order,

European Journal of Applied Mathematics, DOI: https://doi.org/10.1017/S095679251800044X.

TEW, R. H. (2019), Friendlander-Keller ray expansions and scalar wave reflection at canonically perturbed boundaries,

European Journal of Applied Mathematics, 30(1), 1 - 22.

Zauderer, E. (1989), Partial Differential Equations of Applied Mathematics - Second Edition, Polytechnic University (New York), Wiley. 